\title{
Autologous Platelet Lysate Could Have Role in Enhancing the Fracture Healing in Rabbits
}

\section{Vafa Meftahpour}

Tabriz Medical University: Tabriz University of Medical Sciences

\section{Farzaneh Ghorbani}

Tabriz Medical University: Tabriz University of Medical Sciences

\section{Majid Ahmadi}

Tabriz Medical University: Tabriz University of Medical Sciences

\section{Ali Aghebati-Maleki}

Tabriz Medical University: Tabriz University of Medical Sciences

\section{Ali Fotouhi}

Tabriz Medical University: Tabriz University of Medical Sciences

\section{Majid Zamani}

Gonabad University of Medical Sciences

\section{Arash Maleki}

Shahid Beheshti University of Medical Sciences School of Medicine

\section{Mansour Khakpour}

Islamic Azad University

leili Aghebati-Maleki ( $\square$ leili_aghebati_maleki@yahoo.com )

Tabriz Medical University: Tabriz University of Medical Sciences https://orcid.org/0000-0002-00445961

\section{Research Article}

Keywords: Platelet lysate, fracture healing, Cell therapy

Posted Date: May 6th, 2021

DOl: https://doi.org/10.21203/rs.3.rs-471699/v1

License: (9) This work is licensed under a Creative Commons Attribution 4.0 International License. Read Full License 


\section{Abstract}

Although bone substitutes supplemented with platelet derived products are widely used in bone regeneration, the role of Platelet lysate needs to be further elucidate in treatment of bone damages. Accordingly, the aim of this animal study was to evaluate the benefits of autologous PL (APL) in fracture healing. Rabbit APL obtained from freeze-thawed platelet suspension and used for treatment of femoral bone damage in 20 rabbits after surgery procedure. Twenty other rabbits with femoral bone damage were assigned to control group which received Phosphate-buffered saline as therapy. ELISA and real-time PCR used for investigation of the secretion and mRNA expression of IL-1, IL-6, TNF- $\alpha$ and TGF- $\beta$ cytokines, respectively. The mRNA expression of several other related genes including MMP-3, MMP-13, IGF, FGF, VEGF and PDGF was also evaluated in days $0,15,30$ and 60 which was higher in APL-treated rabbits than that of controls. However, secretion of pro-inflammatory cytokines was meaningfully lower than the control group and reduced significantly after two weeks. These observations indicated that factors released from PL and regulation between them could result in bone fracture healing.

\section{Introduction}

The treatment of bone defects represents an important clinical challenge in the management of orthopaedic trauma patients [1]. Bone grafting, especially autografts, are considered as the major surgical procedure in the treatment of bone defects or nonunion fractures, due to their capacity in replacing the missing bone, providing mechanical support and enhancing the biological repair [2, 3]. However, they have their own drawbacks, including limited availability, higher operating time, chronic donor site pain, and extra costs $[4,5]$. Accordingly, development of novel and effective approaches for bone repair and regeneration is of great importance for better treatment in these patients.

Platelets, also called thrombocytes, are cell fragments which participate in angiogenesis, vascular remodeling and tissue repair and regeneration through releasing multiple growth factors and cytokines [2, 6 , 7]. Platelet lysate [8] is a cocktail of growth factors obtained from the lysis of platelets concentrated in a small volume of plasma and has been mainly used as fetal calf serum (FCS) replacement for cell culture $[9,10]$. When compared to other platelet derived products such as platelet-rich plasma (PRP), PL contains higher amounts of cytokines, chemokines and growth factors and its preparation is more costeffective and relatively easier [11-13]. Another advantage is that it can be stored frozen for long time, whereas PRP is thermo-sensitive and cannot be stored under $4^{\circ} \mathrm{C}[14]$.

Several in vitro studies have shown the potential of PL in enhancing wound healing process $[15,16]$. Furthermore, it has already been used in several clinical trials for treatment of different disorders such as diabetic foot ulcers [17], chronic venous ulcers [18], lumbar radicular pain [19], refractory lateral epicondylitis [20], lateral epicondylitis [21], alopecia [22] and Oral mucositis [23]. It has also been suggested that PL could be used as a supplement for proliferation and transplantation of mesenchymal stem cells in Graft versus host disease (GvHD) corticosteroids resistant patients [24, 25]. 
However, the role of PL in bone regeneration has not been properly determined. Therefore, the objective of this study was to study the potential of $\mathrm{PL}$ in fracture healing in rabbits through analyzing the secretion of pro-inflammatory cytokines (IL-1, IL-6, TNF- $a$ ) and transforming growth factor $\beta$ (TGF- $\beta$ ) and their mRNA expression levels. We also decided to measure the mRNA expression levels of bone growth factors including insulin-like growth factor (IGF), fibroblast growth factors (FGF), vascular endothelial growth factor (VEGF), and platelet-derived growth factor (PDGF), and also matrix metalloproteinases-3 (MMP-3) and MMP-13, which involved in the active regulation of bone quality [26]. Ultimately, results achieved in this study may provide novel insights into the involvement of PL in repair and healing of bone fracture.

\section{Methods}

\section{Experimental Model}

Forty 17-week-old male New Zealand rabbits with a mean weight of $3 \mathrm{~kg}$ were used in this study. All procedures were carried out with the approved of the Ethics Committee of Tabriz University of Medical Sciences (IR.TBZMED.VCR.REC.1398.070). The rabbits were housed individually in metal cages at a temperature of $21^{\circ} \mathrm{C}$ and humidity at $50 \%$, and kept on a $12: 12$ light:dark cycle and acclimatized for two weeks before the trial.

\section{Preparation of rabbit Autologous Platelet Lysate (APL)}

A protocol described by Soffer et al. [27] was applied for preparation of APL from the rabbits a week prior to surgery. Briefly, $10 \mathrm{ml}$ of blood samples were obtained from the intermediate branch of the caudal auricular vein of each rabbit and collected in Venoject tubes (3:1 ( $\mathrm{v} / \mathrm{v})$ blood: ACD-A). Then, the tubes were centrifuged at $1500 \times \mathrm{g}\left(10 \mathrm{~min}, 20^{\circ} \mathrm{C}\right)$ to isolate platelet pellets and subsequently, PRP was removed and stored at $-80^{\circ} \mathrm{C}$. The packed platelets were resuspended to $10^{9}$ platelets $/ \mathrm{ml}$ in isotonic saline. After five repeated cycles of freezing thawing, each APL was centrifuged at $10^{4} \times \mathrm{g}\left(1 \mathrm{~h}, 4^{\circ} \mathrm{C}\right)$ to eliminate any derbies and leukocytes. Afterwards, the supernatant was collected and filtered through 0.2 $\mu \mathrm{m}$ filter and stored at $-80^{\circ} \mathrm{C}$ for subsequent analysis.

\section{Surgical Procedure}

The rabbits were anesthetized by intramuscular administration of ketamine (100 mg/ kg), Diazepam (50 $\mathrm{mg} / \mathrm{kg}$ ) and metedomidine $(25 \mathrm{mg} / \mathrm{kg})$ combination in the hind limb. The surgical process was done according to the study conducted by Soffer et al. [27]. A cylinder-shaped critical-size defect (10 mm deep, $5.5 \mathrm{~mm}$ wide) was created in the lateral condyle by using color-coded drills, following a longitudinal skin incision in this site which was thoroughly rinsed with isotonic saline to eliminate bone fragments. Afterwards, the exterior surface of the surgical site was disinfected and closed using skin flap. Then, all the rabbits received intramuscular administration of $0.2 \mathrm{mg} / \mathrm{kg}$ metoxicamn (Metakam Boehringer Ingelheim Vetmedica $\mathrm{GmbH}$, Germany) to reduce the postoperative pain each 24-h period. Prophylactic antibacterial treatment, consisting of sulfadimethoxine trimethoprime at $25 \mathrm{mg} / \mathrm{kg}$ (Copylap Biove', France), was also administered for 5 days after surgery. 


\section{APL administration}

The rabbits were randomly assigned to two control and APL-treated groups, each having 20 animals. In the APL-treated and control groups, the wound site filled with $200 \mu \mathrm{l}$ APL and Phosphate-buffered saline (PBS), respectively.

\section{Enzyme linked immunosorbent assay (ELISA)}

The serum from the APL-treated rabbits and the control group were used for evaluating the concentration of pro-inflammatory (IL-1, IL-6, TNF-a) cytokines and TGF- $\beta$ ( $\mathrm{pg} / \mathrm{ml})$ in days $0,15,30$ and 60 by ELISA in line with the manufacturer's instructions (Mybiosource, San Diego, USA). All samples were assessed in duplicate to improve accuracy. Absorbance values were measured by a microplate reader, and the concentration of cytokines was analyzed according to the standard curves.

\section{RNA extraction, complementary DNA preparation and Real- Time quantitative polymerase chain reaction (RT-qPCR)}

To investigate the effect of APL on fracture healing, we studied the gene expression levels of IL-1, IL-6, TNF- $\alpha$, TGF- $\beta$, MMP-3, MMP-13, IGF, FGF, VEGF and PDGF in the APL-treated rabbits and the control group in days $0,15,30$ and 60 . Total RNA was extracted from the PBMCs using RNX-PLUS Solution (SinaClon, Tehran, Iran) and complementary DNA (cDNA) was synthesized by random hexamer, oligo(dT) primers, and M-MLV (H-) Revert Aid Reverse Transcriptase kit (Thermo Fisher, Waltham, MA). Concentration and purity of RNA content was evaluated by Nano-Drop spectrophotometer (Agilent Technologies, USA). RTqPCR was performed on a Light Cycler 2.0 Real-Time PCR System machine (Roche Applied Science, Germany) using specific forward and reverse primers and SYBR Green dye (Roche, Germany). GAPDH was used as the reference gene for normalization of mRNA expressions. The relative expression of mRNAs was evaluated by the threshold cycle (Ct) value, according to the $2^{-\triangle \triangle C T}$ method in each sample. For amplification confirmation, we used electrophoretic analysis on $2 \%$ agarose gel and the DNA sequencing (SEQLAB, Germany). The primer sequences are presented in Table 1. 
Table 1

Primers for real-time polymerase chain reaction (RT-PCR)

\begin{tabular}{|c|c|c|}
\hline Gene & Primer & Sequence $\left(5^{\prime} \rightarrow 3^{\prime}\right)$ \\
\hline \multirow[t]{2}{*}{ IL-1 } & Forward & GACCTTCCAGGATGAGGACA \\
\hline & Reverse & AGGCCACAGGTATTTTGTCG \\
\hline \multirow[t]{2}{*}{ IL-6 } & Forward & TCTCCAGCAACGAGGAGAAT \\
\hline & Reverse & TGTGATCTGAAACCTGCTGC \\
\hline \multirow[t]{2}{*}{ TNF-a } & Forward & GCTGCACTTCAGGGTGATC \\
\hline & Reverse & CTTGTTCGGGTAGGAGACG \\
\hline \multirow[t]{2}{*}{ TGF- $\beta$} & Forward & GCA AGG ACC TGG GCT GGA A \\
\hline & Reverse & AGT AAC ACG ATG GGC AGT GGC \\
\hline \multirow[t]{2}{*}{ IGF } & Forward & TCT GCG GTG CTG AGC TGG TG \\
\hline & Reverse & TGC CTT TGC CGG CTT GAG GG \\
\hline \multirow[t]{2}{*}{ FGF } & Forward & GGA GAA GAG CGA CCC ACA CAT CA \\
\hline & Reverse & TAG CCT TCT GCC CAG GTC CTG TT \\
\hline \multirow[t]{2}{*}{ VEGF } & Forward & CGC AGC TAC TGC CAG CCG AT \\
\hline & Reverse & GCA CCA GAG GCA CGC AGG AA \\
\hline \multirow[t]{2}{*}{ PDGF } & Forward & TGC ACC GGC TCA TCC TCG TCT A \\
\hline & Reverse & GTC ATC TCG CCG GAG ATT GGC GTT G \\
\hline \multirow[t]{2}{*}{ MMP-3 } & Forward & CTG GAG GTT TGA TGA GAA GA \\
\hline & Reverse & CAG TTC ATG CTC GAG ATT CC \\
\hline \multirow[t]{2}{*}{ MMP-13 } & Forward & GCA GCA GTC TCC AGG CAC GG \\
\hline & Reverse & TCA GGG ACC CCG CAT CTC GG \\
\hline \multirow[t]{2}{*}{ GAPDH } & Forward & TCA CCA TCT TCC AGG AGC GA \\
\hline & Reverse & CAC AAT GCC GAA GTG GTC GT \\
\hline
\end{tabular}

\section{Statistical analysis}

Statistical analysis and drawing the graphs were done using GraphPad Prism (version 9.0.0). The normality of data distribution was assessed using the Kolmogorov-Smirnov test. Also, unpaired $t$ test was applied to compare the quantitative variables between the studied groups. Descriptive data were in the form of mean \pm standard deviation (SD) and $P<0.05$ was reported to be statistically significant. 


\section{Results}

\section{Serum secretion levels of cytokines (IL-1, IL-6, TNF- $a$ and TGF- $\beta$ )}

According to the Fig. 1, the levels of IL-1 cytokine in the serum of the APL-treated rabbits were lower in days $15(P=0.026), 30(P=0.0074)$ and $60(P=0.039)$ compared to the control group. Our results also indicate that IL-1 secretion reduced in days $30(P=0.014)$ and $60(P=0.0073)$ compared with day 15 in the APL-treated group.

Our investigations also showed a significant decrease in the levels of IL- 6 cytokine in days 15 ( $P=$ 0.0012), $30(P<0.0001)$ and $60(P=0.0048)$ in the APL-treated group in comparison with the controls. In addition, we indicated that IL-6 secretion was noticeably lower in day 60 compared with day 15 in the APL-treated rabbits $(P=0.0013)$, but it was not significant in day $30(P=0.054)$. Figure 1 also show a significant increase in IL-6 level in day $15(P<0.0001)$ and $30(P=0.0004)$ as compared with day 0 in the control group. However, it was significantly reduced in day 60 than that of $15(P<0.0001)$.

The level of TNF-a was also lower in the APL-treated group compared with the controls in days 15 ( $P=$ 0.0002), $30(P=0.0078)$ and $60(P=0.013)$. Besides, it was demonstrated that TNF-a secretion was meaningfully decreased in day 60 as compared with day $15(P=0.0078)$ in the APL-treated group. Additionally, the level of this cytokine was increased in day $15(P=0.0008)$ than that of day 0 and then it was significantly decreased in day $30(P=0.0038)$ and $60(P<0.0001)$ in comparison with day 15 in the control group.

In contrast, it was revealed that the serum levels of TGF- $\beta$ was considerably increased in rabbits treated with APL in days $15(P=0.0001)$ and $30(P<0.0001)$ as compared with the control group, but the difference was not statistically significant in day $60(P=0.409)$. Our data also showed that the level of TGF- $\beta$ secretion was noticeably increased in APL-treated group in days $15(P=0.0003), 30(P=0.0001)$ and $60(P=0.029)$ than that of day 0 . Furthermore, no significant change was found in the control group in day $15(P=0.834)$ in comparison with day 0 , but it was elevated in days 30 and $60(P=0.028$ and $P=$ 0.027 , respectively).

\section{mRNA expression level of cytokines (IL-1, IL-6, TNF- $a$ and TGF- $\beta$ ) and other related genes (MMP-3, MMP-13, IGF, FGF, VEGF and PDGF)}

IL-1, IL-6, TNF- $\alpha$, TGF- $\beta$, MMP-3, MMP-13, IGF, FGF, VEGF and PDGF mRNA expressions were evaluated in the PBMCs obtained from APL-treated and untreated rabbits in days $0,15,30$ and 60 . 
As demonstrated in Fig. 2, a significant reduction was found in the level of IL-1 in days 15 and 30 between the APL-treated and control group $(P=0.023$ and $P=0.026$, respectively). Furthermore, in the APL-treated group, this cytokine was considerably decreased between days 15 to $60(P<0.0001)$. We also showed in Fig. 2 that IL-6 level was lower in the APL-treated group in comparison with the control in day $15(P=0.0003)$ and day $30(P=0.032)$. The mRNA expression levels of TNF-a also recognizably reduced in APL-treated rabbits compared to the controls in days $15(P=0.0062), 30(P=0.0028)$ and $60(P=$ 0.0006). Moreover, we showed that the decrease between the mRNA expression levels of this cytokine was significant in the APL-treated group between days 15 to $30(P=0.0048)$ and $60(P<0.0001)$. As demonstrated in Fig. 2 , the expression levels of TGF- $\beta$ was also higher in the APL-treated group than that of controls in days $15(P=0.0001)$ and $30(P=0.0001)$ and $60(P=0.04)$. Our results also indicated that this growth factor meaningfully increased from day 0 to $15(P=0.008), 30(P<0.0001)$ and $60(P=$ 0.0003).

Furthermore, the mRNA expression levels of MMP-3 were elevated in the APL-treated group as compared with the control group in days $15(P<0.0001), 30(P<0.0001)$ and $60(P<0.0001)(F i g .3)$ and the expression of MMP-13 was also higher in the treated rabbits in days $15(P<0.0001)$ and $30(P<0.0001)$.

Results from this study also indicated that the expression level of IGF, FGF, VEGF and PDGF increased significantly from day 0 to $15(P=0.0001, P=0.0054, P=0.002$ and $P=0.027$, respectively). Besides, their level was considerably elevated from day 0 to $30(P<0.0001, P<0.0001, P=0.0002$ and $P<0.0007$, respectively) and 0 to $60(P=0.0003, P<0.0001, P=0.0004$ and $P<0.0005$, respectively, Fig. 4).

\section{Discussion}

Among various procedures which have been applied for regeneration or repair of bone damages or fracture healing, platelet derived products such as PLs have achieved notable attention because of high amounts of cytokines, chemokines and growth factors as well as easy preparation and low costs [28]. Currently, relatively little is known about the role of APL in bone fracture healing.

Several other studies have also examined the efficacy of PL on bone repair or formation. In two studies conducted by Chakar et al., the impact of APL was evaluated on regeneration of rabbit's femoral and skull bone $[29,30]$. They suggested that APL could have beneficial role on vertical bone formation and regeneration. Besides, it was shown that PL in combination with Biphasic calcium phosphate or demineralised bovine bone mineral do not provide any additional effect. In another investigation, Soffer et al. (2006) found that APL, in combination with calcium carbonate ceramic particles could elevate resorption and bone formation in both femurs and calvaria bones in rabbit models [27]. Babo, Carvalho, et al. (2016) also assessed the potential of PL integrated with calcium phosphate cement increased cement on bone formation in rats with calvarial defects. According to the results, no significant changes were found in the absence of PL [31]. It has also been demonstrated that human PL coating on scaffolds combined with mesenchymal stem cells, could enhance neovascularization and bone formation [32]. In another study conducted by Centeno et al. (2017), PL was used as a promising choice for treatment of 
lumbar radicular pain [33]. It has also been observed that APL percutaneous injection could be a new therapeutic candidate for the treatment of non-union or delayed healing fractures [34].

According to the evidence, PL-derived cytokines, growth factors and adherence molecules could result in bone regeneration through differentiation, proliferation, angiogenesis and migration of cells to the inflammatory site [28]. Therefore, in this study we investigated the impact of APL in fracture healing in rabbits through evaluating of the pro-inflammatory cytokines including IL-1, IL- 6 and TNF-a. Our results indicated that the level of these cytokines was significantly lower in APL-treated group than that of the controls. In accordance with these findings, we illustrated that the m-RNA expression level of IL-6 and TNF-a was also decreased after 2 weeks in APL-treated rabbits in comparison with the control group. TGF- $\beta$ is an anti-inflammatory cytokine involved in osteoblasts differentiation, migration and promotion of angiogenesis along with other platelet factors [35]. According to the results of this study, a significant elevation in secretion and m-RNA expression of TGF- $\beta$ were found in APL-treated rabbits compared with the controls. Thus, our results support the concept that regulation between anti- and pro-inflammatory cytokines released from APL may result in bone regeneration.

IGF, FGF, VEGF and PDGF are other PL growth factors which are essential for bone regeneration and have synergistic and proper effects when combined together [28]. Our study also found that these factors considerably increased after 60 days in APL-treated group. MMP-3 and MMP-13 are two other PL factors which act as regulator of bone quality, considerably increased in APL-treated rabbits up to day 60 .

\section{Conclusion}

Taken together, the results of this study proposed that APL can possess essential role in bone fracture healing. We showed that decreased levels pro-inflammatory cytokines such as IL-1, IL-6 and TNF- $\alpha$ and up-regulation of as anti-inflammatory cytokine (TGF- $\beta$ ) can result in bone fracture healing. Furthermore, our findings indicate a notable increase in the levels of growth factors including IGF, FGF, VEGF and PDGF and MMP-3 and MMP-13 which are of great importance in bone regeneration. Basically, results archived in our study provide a novel treatment opportunity for bone fracture healing.

\section{Abbreviations}

CDNA

complementary DNA; ELISA:Enzyme linked immunosorbent assay; FCS:Fetal calf serum; FGF:Fibroblast growth factors; IGF:Insulin-like growth factor; MMP-3:Matrix metalloproteinases-3; PBS:Phosphatebuffered saline; PDGF:Platelet-derived growth factor; PRP:Platelet-rich plasma; RT-qPCR:Real-Time quantitative polymerase chain reaction; TGF- $\beta$ :Transforming growth factor $\beta$; VEGF:Vascular endothelial growth factor.

\section{Declarations}




\section{Acknowledgments}

The authors would like to thank all the participants for contributing to the accomplishment of the present study. Financial support to the present study was provided by Research Vice- Chancellor at Tabriz University of Medical Sciences, Iran [Grant No. 62058].

\section{Authors' contributions}

Vafa Meftahpour and Farzaneh Ghorbani have made contributions to the writing of the manuscript. Majid Ahmadi and Ali Aghebati-Maleki performed the experiments and wrote the manuscript supervised by Ali Fotouhi, Arash Maleki who edited the manuscript. Majid Zamani and Mansour Khakpour performed the data interpretation and manuscript editing by leili Aghebati-Maleki. All authors read and approved the final manuscript.

\section{Funding}

This study was supported by the Stem Cell Research Center (SCRC), Tabriz University of Medical Sciences, Tabriz, Iran. [Grant No. 62058].

\section{Availability of data and materials}

The datasets used and/or analyzed during the current study are available from the corresponding author on reasonable request.

\section{Ethics approval and consent to participate}

All procedures were carried out with the approved of the Ethics Committee of Tabriz University of Medical Sciences (IR.TBZMED.VCR.REC.1398.070).

\section{Consent for publication}

Not applicable

\section{Competing interests}

The authors declare that they have no competing interests.

\section{References}

1. Guerado, E. and E. Caso, Challenges of bone tissue engineering in orthopaedic patients. World Journal of Orthopedics, 2017. 8: p. 87.

2. Zhang, N., et al., Research Progress in the Mechanism of Effect of PRP in Bone Deficiency Healing. The Scientific World Journal, 2013. 2013: p. 134582. 
3. Fotouhi, A., et al., Platelet rich plasma, stromal vascular fraction and autologous conditioned serum in treatment of knee osteoarthritis. Biomedicine and Pharmacotherapy, 2018. 104: p. 652-660.

4. Pieske, 0. , et al., Autologous bone graft versus demineralized bone matrix in internal fixation of ununited long bones. Journal of Trauma Management \& Outcomes, 2009. 3(1): p. 11.

5. Roffi, A., et al., Platelet-rich plasma for the treatment of bone defects: from pre-clinical rational to evidence in the clinical practice. A systematic review. International Orthopaedics, 2017. 41(2): p. 221237.

6. Burnouf, T., et al., Human platelet lysate: Replacing fetal bovine serum as a gold standard for human cell propagation? Biomaterials, 2016. 76: p. 371-387.

7. Burnouf, T., et al., Platelet microparticles: detection and assessment of their paradoxical functional roles in disease and regenerative medicine. Blood reviews, 2014. 28(4): p. 155-166.

8. Avanzini, M.A., et al., Humoral immunodeficiencies in Down syndrome: serum IgG subclass and antibody response to hepatitis B vaccine. Am J Med Genet Suppl, 1990. 7: p. 231-3.

9. Notodihardjo, S.C., et al., Comparison of the efficacy of cryopreserved human platelet lysate and refrigerated lyophilized human platelet lysate for wound healing. Regenerative Therapy, 2019. 10: p. 1-9.

10. Nguyen, V.T., et al., Platelet Lysate Induces in Human Osteoblasts Resumption of Cell Proliferation and Activation of Pathways Relevant for Revascularization and Regeneration of Damaged Bone. International Journal of Molecular Sciences, 2020. 21(14).

11. Hesler, M., et al., Non-pooled Human Platelet Lysate: A Potential Serum Alternative for In Vitro Cell Culture. Alternatives to Laboratory Animals, 2019. 47(3-4): p. 116-127.

12. Bari, E., et al., Association of silk sericin and platelet lysate: Premises for the formulation of wound healing active medications. International Journal of Biological Macromolecules, 2018. 119.

13. Meftahpour, V., et al., Platelet lysate: A promising candidate in regenerative medicine. Regenerative Medicine, 2021. 16(1): p. 71-85.

14. del fante, C., et al., Platelet Lysate Mucohadesive Formulation to Treat Oral Mucositis in Graft Versus Host Disease Patients: A New Therapeutic Approach. AAPS PharmSciTech, 2011. 12: p. 893-9.

15. Backly, R.E., et al., Platelet Lysate Induces In Vitro Wound Healing of Human Keratinocytes Associated with a Strong Proinflammatory Response. Tissue Engineering Part A, 2011. 17(13-14): p. 1787-1800.

16. Ranzato, E., et al., Platelet lysate promotes in vitro wound scratch closure of human dermal fibroblasts: different roles of cell calcium, P38, ERK and PI3K/AKT. Journal of Cellular and Molecular Medicine, 2009. 13(8b): p. 2030-2038.

17. Jafar, H., et al., Platelet lysate promotes the healing of long-standing diabetic foot ulcers: A report of two cases and in vitro study. Heliyon, 2020. 6(5): p. e03929.

18. Stacey, M.C., et al., Randomised Double-blind Placebo Controlled Trial of Topical Autologous Platelet Lysate in Venous Ulcer Healing. European Journal of Vascular and Endovascular Surgery, 2000. 
20(3): p. 296-301.

19. Centeno, C., et al., The use of lumbar epidural injection of platelet lysate for treatment of radicular pain. Journal of Experimental Orthopaedics, 2017. 4(1): p. 38.

20. Tan, X.-X., et al., Autologous platelet lysate local injections for the treatment of refractory lateral epicondylitis. Journal of Orthopaedic Surgery and Research, 2016. 11(1): p. 17.

21. Tan, X.-X., et al. Autologous platelet lysate local injections for the treatment of refractory lateral epicondylitis. Journal of orthopaedic surgery and research, 2016. 11, 17 DOI: 10.1186/s13018-0160349-2.

22. Cole, J.P., et al., Alopecia and platelet-derived therapies. Stem Cell Investigation, 2017. 4(11).

23. Del Fante, C., et al., Platelet lysate mucohadesive formulation to treat oral mucositis in graft versus host disease patients: a new therapeutic approach. AAPS PharmSciTech, 2011. 12(3): p. 893-9.

24. Introna, M., et al., Treatment of Graft versus Host Disease with Mesenchymal Stromal Cells: A Phase I Study on 40 Adult and Pediatric Patients. Biology of Blood and Marrow Transplantation, 2014. 20(3): p. 375-381.

25. Lucchini, G., et al., Platelet-lysate-Expanded Mesenchymal Stromal Cells as a Salvage Therapy for Severe Resistant Graft-versus-Host Disease in a Pediatric Population. Biology of Blood and Marrow Transplantation, 2010. 16(9): p. 1293-1301.

26. Tang, S.Y., et al., Matrix metalloproteinase-13 is required for osteocytic perilacunar remodeling and maintains bone fracture resistance. Journal of bone and mineral research : the official journal of the American Society for Bone and Mineral Research, 2012. 27(9): p. 1936-1950.

27. Soffer, E., et al., Effects of autologous platelet lysates on ceramic particle resorption and new bone formation in critical size defects: The role of anatomical sites. Journal of Biomedical Materials Research Part B: Applied Biomaterials, 2006. 79B(1): p. 86-94.

28. Zamani, M., et al., Novel therapeutic approaches in utilizing platelet lysate in regenerative medicine: Are we ready for clinical use? Journal of Cellular Physiology, 2019. 234(10): p. 17172-17186.

29. Chakar, C., et al., Bone Formation with Deproteinized Bovine Bone Mineral or Biphasic Calcium Phosphate in the Presence of Autologous Platelet Lysate: Comparative Investigation in Rabbit. International Journal of Biomaterials, 2014. 2014: p. 367265.

30. Chakar, C., et al., Vertical bone regeneration with deproteinised bovine bone mineral or biphasic calcium phosphate in the rabbit calvarium: effect of autologous platelet lysate. Journal of Materials Science: Materials in Medicine, 2015. 26(1): p. 23.

31. Babo, P.S., et al., Assessment of bone healing ability of calcium phosphate cements loaded with platelet lysate in rat calvarial defects. Journal of Biomaterials Applications, 2016. 31(5): p. 637-649.

32. Leotot, J., et al., Platelet lysate coating on scaffolds directly and indirectly enhances cell migration, improving bone and blood vessel formation. Acta biomaterialia, 2013. 9(5): p. 6630-6640.

33. Centeno, C., et al., The use of lumbar epidural injection of platelet lysate for treatment of radicular pain. Journal of Experimental Orthopaedics, 2017. 4. 
34. Jiang, H.j., et al., Autologous platelet lysates local injections for treatment of tibia non-union with breakage of the nickelclad: a case report. SpringerPlus, 2016. 5.

35. Tan, C., et al., Feasibility and Efficiency of Human Bone Marrow Stromal Cell Culture with Allogeneic Platelet Lysate-Supplementation for Cell Therapy against Stroke. Stem Cells International, 2016. 2016: p. 6104780.

\section{Figures}
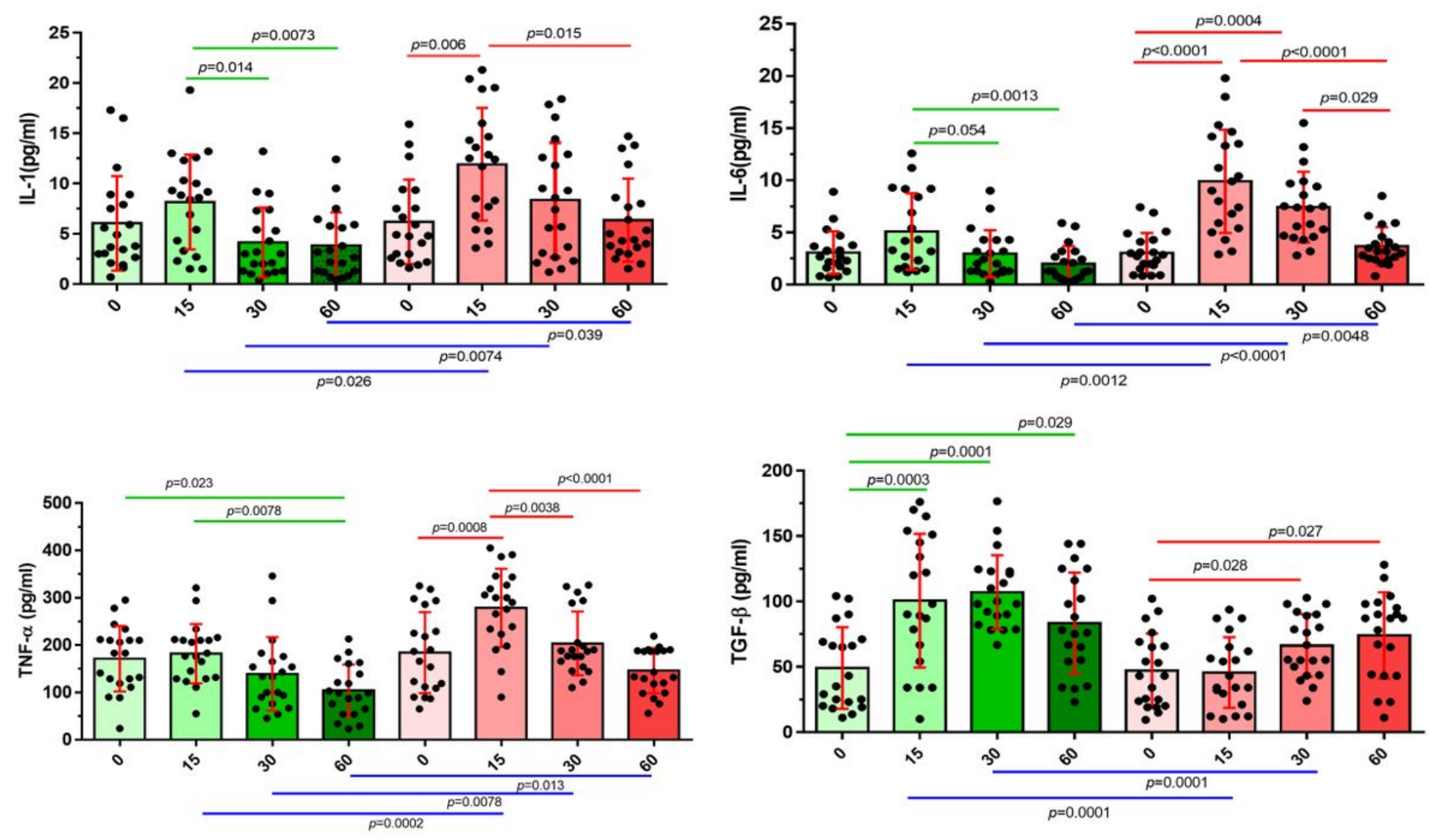

\section{Figure 1}

Serum secretion levels of cytokines (IL-1, IL-6, TNF- $\alpha$ and TGF- $\beta$ ) in APL-treated rabbits in comparison with the control group in days $0,15,30$ and 60 . The secretion of IL-1 was significantly lower in APLtreated rabbits in days $15(P=0.026), 30(P=0.0074)$ and $60(P=0.039)$. The secretion of IL-6 was also significantly lower in these rabbits in days $15(P=0.0012), 30(P<0.0001)$ and $60(P=0.0048)$ and TNF-a secretion was reduced in APL-treated rabbits than that of controls in days $15(P=0.0002), 30(P=0.0078)$ and $60(P=0.013)$. $d)$ However, TGF- $\beta$ showed an increase in days $15(P=0.0001)$ and $30(P=0.0001)$ in rabbits treated with APL. Provided as mean $\pm S D$ (APL-treated rabbits, $n=20$, control group, $n=20$ ), $p<$ 0.05 was regarded as statistically meaningful in the results. 


\section{MMP-3}

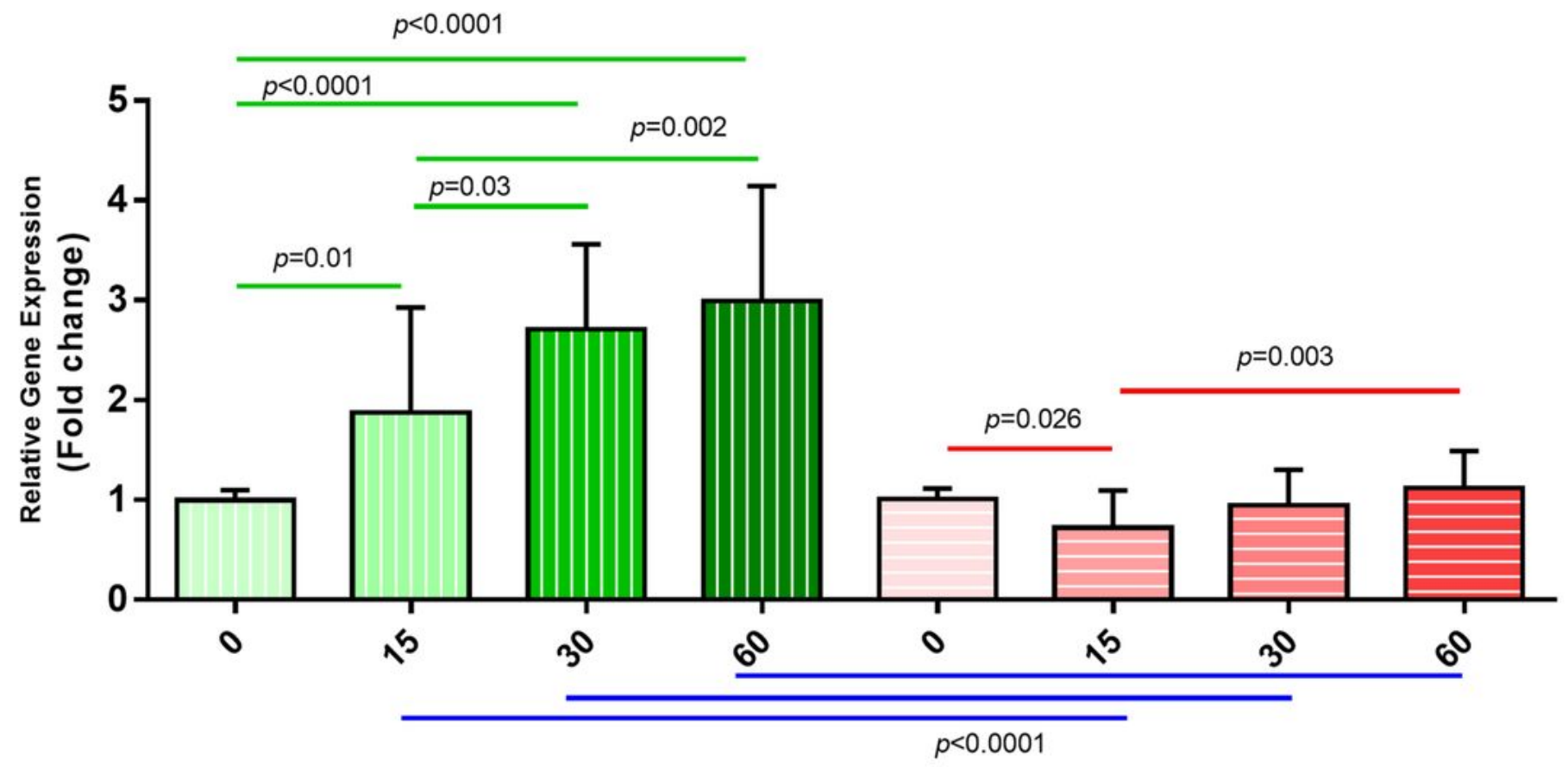

MMP-13

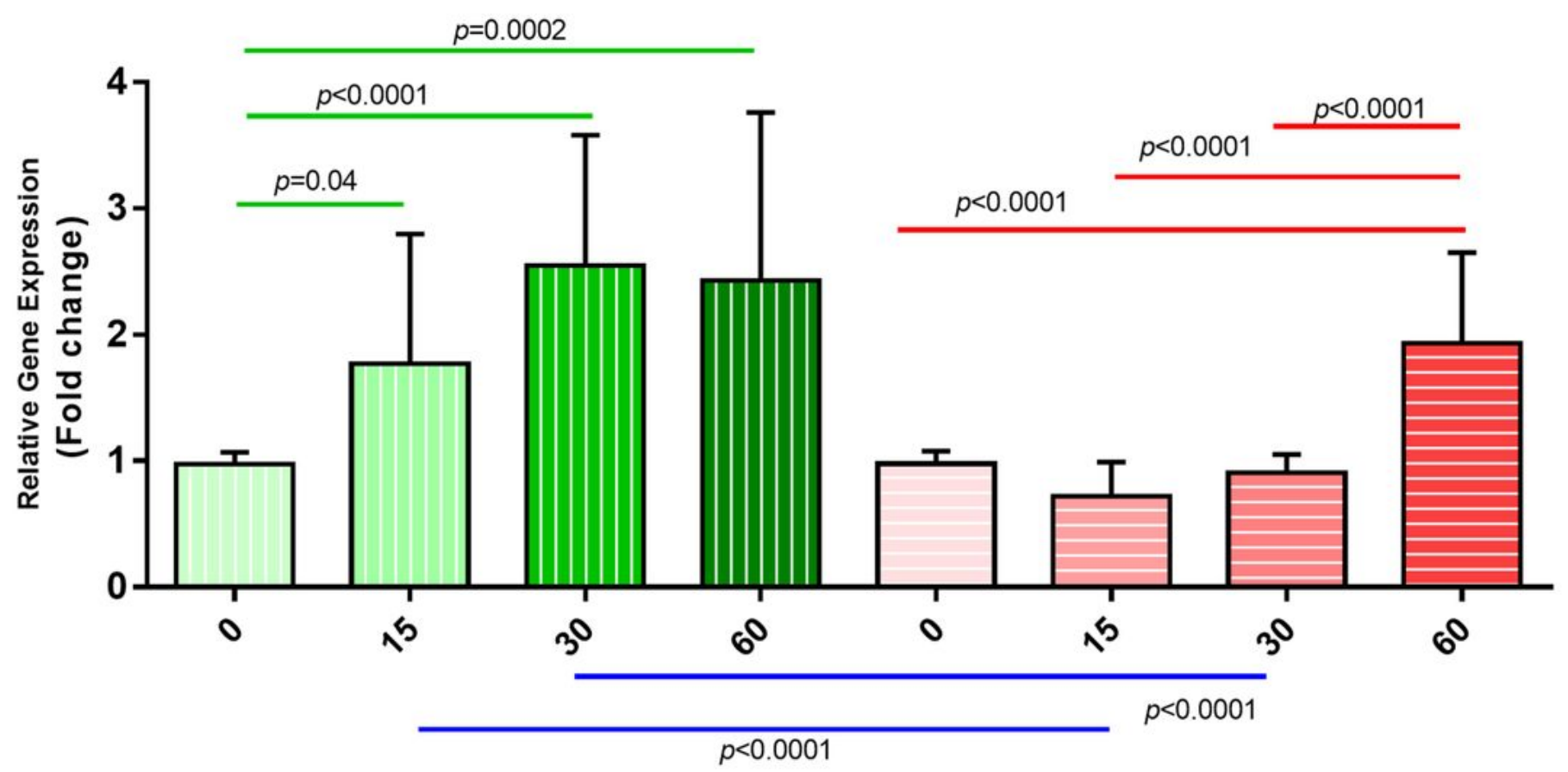

Figure 2

mRNA expression level of cytokines (IL-1, IL-6, TNF- $\alpha$ and TGF- $\beta$ ) in APL-treated rabbits in comparison with the control group in days $0,15,30$ and 60 . The mRNA expression of IL-1 was lower in APL-treated rabbits in days $15(P=0.023)$ and $30(P=0.026)$. The mRNA expression of IL-6 was also significantly lower in these rabbits in days $15(P=0.0003)$ and $30(P=0.032)$ and TNF- $a$ expression was reduced in APL-treated rabbits than that of controls in days $15(P=0.0062)$ and $30(P=0.0028)$ and $60(P=0.0006)$. 
d) However, TGF- $\beta$ showed a significant elevation in days $15(P=0.003), 30(P<0.0001)$ and $60(P=0.04)$ in rabbits treated with APL. Provided as mean $\pm S D$ (APL-treated rabbits, $n=20$, control group, $n=20), p<$ 0.05 was regarded as statistically meaningful in the results.
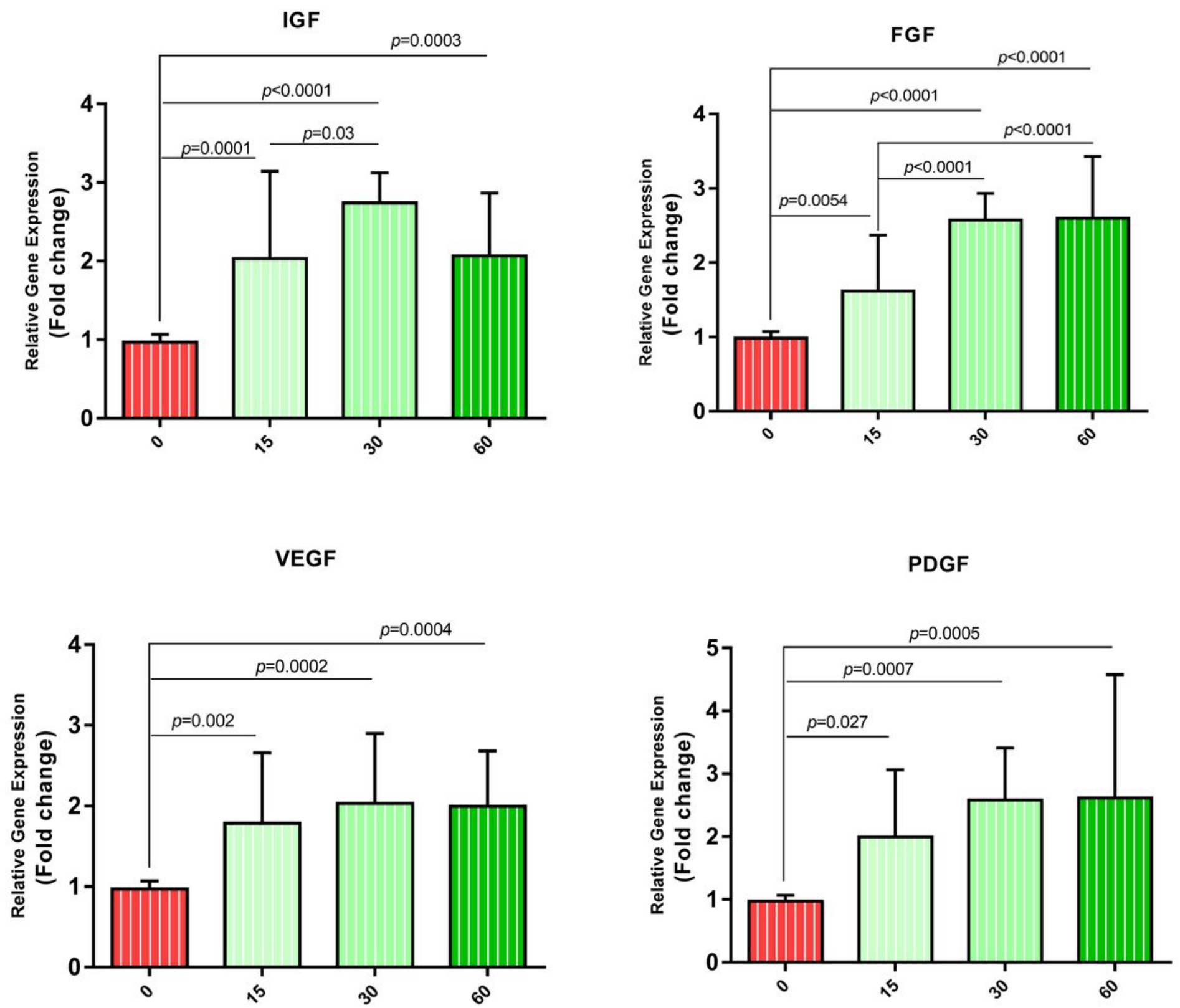

Figure 3

mRNA expression level of MMP-3 and MMP-13 in APL-treated rabbits in comparison with the control group in days $0,15,30$ and 60 . The mRNA expression of MMP-3 and MMP-13 was higher in the APLtreated rabbits as compared with the control group in days 15, 30 and $60(P<0.0001)$. 
IL-1

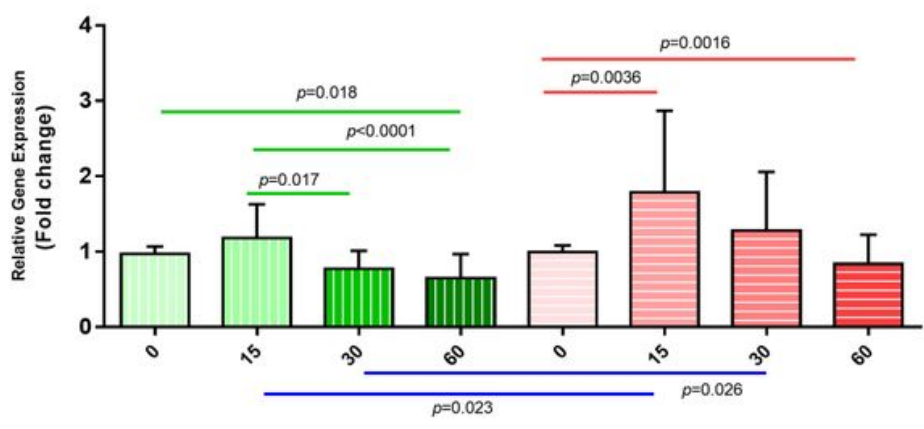

TNF- $\alpha$

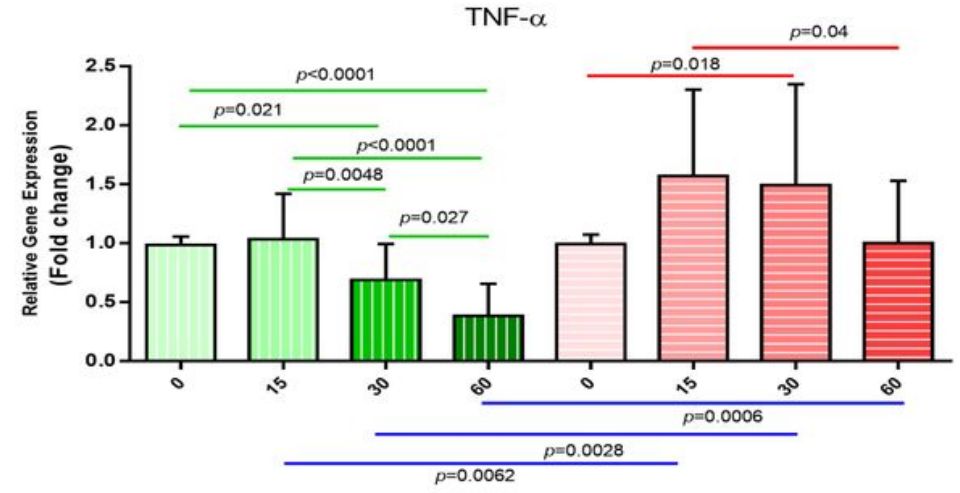

IL-6
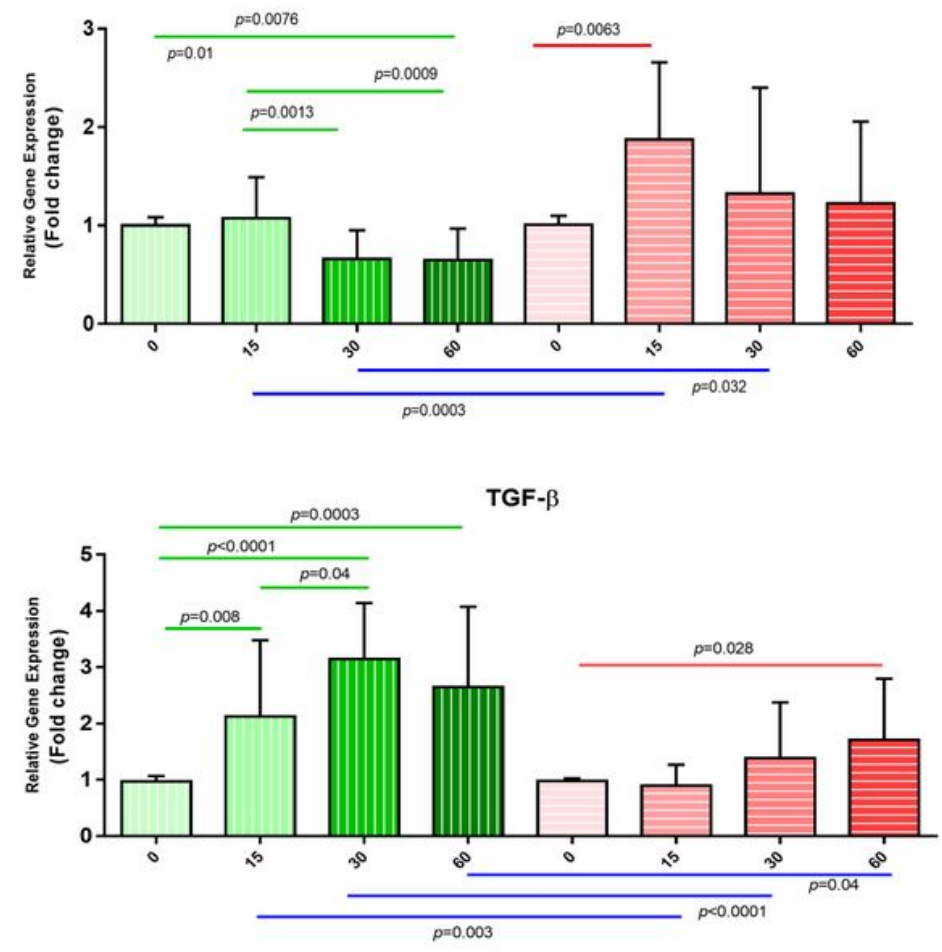

\section{Figure 4}

mRNA expression level of IGF, FGF, VEGF and PDGF in APL-treated rabbits in days 0, 15, 30 and 60 . The expression level of IGF, FGF, VEGF and PDGF elevated after 15 days $(P=0.0001, P=0.0054, P=0.002$ and $P=0.027$, respectively). Besides, their level was noticeably increased from day 0 to $30(P<0.0001, P$ $<0.0001, \mathrm{P}=0.0002$ and $\mathrm{P}<0.0007$, respectively $)$ and 0 to $60(\mathrm{P}=0.0003, \mathrm{P}<0.0001, \mathrm{P}=0.0004$ and $\mathrm{P}<$ 0.0005 , respectively). Provided as mean $\pm S D$ (APL-treated rabbits, $n=20$, control group, $n=20$ ), $p<0.05$ was regarded as statistically meaningful in the results. 